\title{
Uso de fármacos durante el embarazo
}

\author{
José Sandoval Paredes ${ }^{1,2,3, a}$, Cindy Sandoval Paz ${ }^{b}$
}

RESUMEN

La información sobre seguridad de fármacos en la gestación es abundante, pero existe poca evidencia científica, por razones éticas muy pocos ensayos clínicos se han realizado y publicado. Todo esto ha traído como consecuencia que se sobredimensione el peligro de los fármacos en el embarazo, limitando su uso razonable en enfermedades crónicas de gestantes, y, en el otro extremo, que se prescriba la medicación libremente desconociendo el verdadero riesgo que implica administrarlos en el momento y en la dosis inadecuada.

La presente revisión describe la manera en que los cambios fisiológicos en el embarazo modifican considerablemente la farmacocinética de la medicación. Se evalúa el verdadero riesgo potencial de teratogénesis de los fármacos, los criterios específicos para considerarlos como factor etiológico. Se comenta la clasificación de la Administración de Alimentos y Medicamentos estadounidense (FDA, por sus siglas en inglés), los criterios de su clasificación, sus limitaciones, su limitada vigencia actual y la propuesta de nuevas reglas para etiquetar los fármacos en donde considera evaluación clínica, exposición inadvertida y riesgo disponible.

Se realiza la descripción de los riesgos de la medicación de más frecuente uso en las patologías más comunes en el embarazo. Se comenta la correcta manera de informar a la paciente expuesta a supuestos teratógenos, con la finalidad de no afectar la percepción del riesgo.

Hay que considerar que los fármacos prescritos con mayor frecuencia se pueden administrar con relativa seguridad durante el embarazo, indicando los que sean estrictamente necesarios, restringiéndolos en el primer trimestre, prefiriendo los de seguridad comprobada, utilizando la menor dosis eficaz durante el menor tiempo posible, evitando utilizar varios fármacos y vigilando sus efectos colaterales y complicaciones.

Palabras clave: Embarazo; fármacos; teratógenos; categoría de riesgo (Fuente: DeCS BIREME).

\section{Drug use during pregnancy}

\section{ABSTRACT}

There is plenty of information about drug safety during pregnancy, but there is little scientific evidence on this topic. Due to ethical reasons, very scarce clinical assays have been conducted and published. This situation has resulted in the exaggeration of drug risk during pregnancy, thus limiting its reasonable use for chronic diseases in pregnant women. At the other extreme, it has resulted in the careless prescription of drugs not acknowledging the real risk involved in administering them in the inappropriate dose and moment.

This review article describes how physiological changes during pregnancy modify significantly the pharmacokinetics of drugs. The real potential risk of drug-induced teratogenesis and the specific criteria for considering them an etiological factor are evaluated. The U.S. Food and Drug Administration (FDA) pregnancy categories' criteria, limitations, and limited current validity are discussed, as well as its proposal of new regulations on drug labeling, considering clinical evaluations, unnoticed exposure, and risks.

The risks associated with the most frequently used drugs for the most common diseases during pregnancy are described. The correct way to tell the patient exposed to suspected teratogens is discussed, in order to avoid affecting risk perception.

It should be considered that the administration of the most frequently prescribed drugs is relatively safe during pregnancy. Nevertheless, only those strictly necessary must be prescribed, their consumption must be limited during the first trimester, those proven safety must be preferred, the minimum effective dose during the shortest time possible must be used, the administration of several drugs must be avoided, and side effects and complications must be monitored.

Keywords: Pregnancy; drugs; teratogens; risk category (Source: MeSH NLM).

1. Hospital Nacional Arzobispo Loayza. Lima, Perú.

2. Universidad de San Martin de Porres, Facultad de Medicina Humana. Lima, Perú.

3. Universidad Mayor de San Marcos. Lima, Perú.

a. Doctor en Medicina, Magíster en Obstetricia, Ginecólogo Obstetra.

b. Médica Serumista 


\section{INTRODUCCIÓN}

La obstetricia moderna orienta esfuerzos e implementa novedosas estrategias, no solamente para garantizar la salud materna, sino también para tener un recién nacido saludable en el extremo de sus posibilidades. La morbimortalidad neonatal tiene un gran componente en los defectos genéticos que deben ser comprendidos en su justa dimensión. Aproximadamente en el $3 \%$ de los recién nacidos se diagnostica una o más malformaciones morfológicas. En los 5 primeros años de vida se descubre otro $3 \%$ con alguna alteración morfológica. A los 18 años se ha descubierto que 8 a $10 \%$ tienen una o más anormalidades funcionales. En aproximadamente el $65 \%$ no se conoce la causa ${ }^{(1,2)}$, se atribuye a factores ocasionados por la ingesta de fármacos solo en 1 a $5 \%$ de estos casos.

Actualmente es muy preocupante el alto uso de medicamentos en el embarazo. El $5 \%$ de las gestantes sufren de alguna enfermedad crónica preconcepcional (asma, hipertensión arterial crónica, diabetes, enfermedades tiroideas, gastrointestinales, etc.), por lo cual deben continuar con algún tratamiento farmacológico. Registros de la OMS indican que aproximadamente el $85 \%$ de las gestantes toman uno o más fármacos durante el embarazo y un regular porcentaje lo hace sin prescripción médica ${ }^{(3)}$. En nuestra realidad la automedicación es una preocupación constante en la medida que no sólo ingieren medicamentos de dudosa procedencia sino también medicina alternativa, hierbas, raíces, jugos- a quienes les atribuyen propiedades curativas, sin ninguna evidencia científica y con riesgo de efectos deletéreos para la madre y su hijo.

La información sobre seguridad de fármacos en la gestación es abundante, pero la mayoría de investigaciones publicadas son estudios caso-control, registro de la exposición de pacientes a determinados fármacos o ensayos clínicos realizados con animales. La mejor evidencia científica debería proceder de ensayos clínicos en seres humanos, pero por razones éticas es muy limitada su producción ${ }^{(4)}$.

\section{Cambios fisiológicos y farmacocinéticos durante el embarazo}

La placenta no es realmente una "barrera" debido que a través de ella se transfieren fácil y rápidamente muchos nutrientes, fármacos, drogas e inclusive tóxicos. La difusión hacia los tejidos fetales de cualquier fármaco depende de varios factores relacionados con la solubilidad a los lípidos, la fijación a las proteínas, el peso molecular, el grado de ionización y el metabolismo placentario. Cuanto mayor es la edad gestacional mayor es la permeabilidad placentaria. Los cambios fisiológicos que ocurren en el embarazo pueden modificar los cambios de concentración del fármaco, en la farmacocinética, es decir en la absorción, distribución, metabolismo y excreción del fármaco ${ }^{(5)}$.
Absorción: En el embarazo hay una disminución de la motilidad intestinal y una prolongación del tránsito, por acción de la progesterona, esto lleva a una mayor tasa de absorción del fármaco. A nivel respiratorio, en la gestación aumenta el flujo sanguíneo pulmonar, se produce una hiperventilación ocasionada por la taquipnea fisiológica, lo cual conduce que fármacos inhalatorios, por ejemplo, aumenten su absorción ${ }^{(6)}$.

Distribución: En la gestante hay una disminución en las concentraciones máximas del fármaco y un retardo en su eliminación, esto sucede porque hay un aumento del volumen plasmático que origina una disminución en la concentración del fármaco que se administra. La proteinuria fisiológica conduce a hipoproteinemia, que lleva a una baja fijación a proteínas, de esta manera aumenta la fracción libre de los fármacos que finalmente es la activa farmacológica y toxicológicamente.

Metabolización: La progesterona, característica en la gestación, provoca una mayor actividad enzimática de los fármacos, la vida media y su acción disminuye porque aumenta la velocidad de su metabolismo ${ }^{(6)}$.

Excreción: En la gestación aumenta el flujo plasmático renal y la filtración glomerular, lo que lleva a una mayor excreción. Un aumento de la cantidad de medicamento excretado, resulta en una disminución de su concentración plasmática y de su vida media.

\section{El riesgo potencial de teratogénesis}

Los defectos congénitos inducidos por fármacos y otras sustancias químicas representan aproximadamente el $1 \%$ de todos los defectos congénitos ${ }^{(1)}$.

Antes de establecer el factor etiológico en un defecto congénito se deben considerar criterios específicos basados en 3 principios:

1.El defecto congénito debe caracterizarse por completo como producido por la droga. Ejemplo, el labio y paladar hendidos se relacionan con la hidantoína pero también con más de 200 causas genéticas ${ }^{(6)}$.

2.El agente debe cruzar la placenta, y hacerlo de manera suficiente para influir de manera directa en el desarrollo fetal.

3. La exposición a la droga debe ocurrir en un periodo importante del desarrollo:

a.Periodo preimplantacional: El suceso es radical, el embrión se mantiene totalmente íntegro o se produce la muerte y el subsecuente aborto, se le conoce como el periodo de "todo o nada". 
b.Periodo de organogénesis: Ocurre entre la segunda y la octava semana de gestación. Es el período embrionario y el más susceptible a sufrir los efectos de un fármaco teratogénico, se pueden originar malformaciones estructurales importantes que pueden ser incompatibles con el desarrollo de la vida fetal y extrauterina.

c. Periodo fetal: Comprende a partir de la novena semana, las alteraciones morfológicas que se pueden producir con la exposición son menos graves que en la organogénesis pero si se pueden originar alteraciones importantes en el crecimiento y desarrollo funcional del feto ${ }^{(7,8)}$.

Es necesario tomar la información sobre el tema, con ponderación. Se han publicado informes no científicos y tendenciosos desfavorables sobre la seguridad de fármacos que son de uso frecuente y muy útiles en la prescripción diaria. La investigación seria sobre los mismos ha logrado reponer en el mercado estos productos con el consiguiente beneficio de los pacientes ${ }^{(9)}$.

De la misma manera, investigación procedente de estudios con animales no garantiza su inocuidad en el ser humano, el caso más ilustrativo es el de la talidomida, un fármaco sedante e hipnótico que se empezó a comercializar en el año 1958 para contrarrestar las náuseas y vómitos de las gestantes en los primeros tres meses de embarazo, hasta que en 1962 se publica la relación que tenía el uso de esta droga y la aparición de malformaciones en brazos y antebrazos (focomelia o amelia) en niños expuestos. En estos momentos se dejó de pensar en la placenta como una barrera impenetrable ${ }^{(7,8)}$.

Hay sólo un número limitado de medicamentos que han demostrado ser teratogénicos en humanos. De igual manera, hay pocos fármacos que sin duda han sido probados para ser seguros, por lo tanto, es prudente reducir al mínimo el número de medicamentos que se toma y limitar el uso de medicamentos para situaciones en las que el beneficio supere claramente el riesgo; se debe elegir medicamentos con el mejor perfil de seguridad, y utilizarlos en la dosis más baja y durante el menor tiempo que sea eficaz ${ }^{(4)}$.

\section{Clasificación de la FDA}

Con la finalidad de orientar al médico en su decisión de usar los fármacos en una gestante, y en respuesta a la tragedia provocada por la talidomida en el año de 1979 (10), la Food and Drug Administration (FDA) asignó las categorías de riesgo de embarazo (A, B, C, D y X) a todos los fármacos disponibles en los Estados Unidos (Tabla 1).

Tabla 1. Seguridad de fármacos en el embarazo. Clasificación de la FDA

\begin{tabular}{|c|c|c|c|}
\hline Categoria & Seguridad & Descripción & Fármacos de uso frecuente \\
\hline Categoría A & $\begin{array}{l}\text { Estudios controlados } \\
\text { no han demostrado } \\
\text { riesgos }\end{array}$ & $\begin{array}{l}\text { Estudios adecuados en embarazadas } \\
\text { no han demostrado riesgo para el } \\
\text { feto durante el primer trimestre, y } \\
\text { tampoco hay evidencia de riesgo en } \\
\text { el resto del embarazo. Son remotas } \\
\text { las posibilidades de daño fetal. }\end{array}$ & $\begin{array}{l}\text { Ácido fólico, hidróxido de aluminio, } \\
\text { hidróxido de magnesio, sulfato } \\
\text { ferroso, Vit. B1, B12, B6, C; (Vit. A es } \\
\text { categoría A pero en altas dosis es X). }\end{array}$ \\
\hline Categoría B & $\begin{array}{l}\text { No hay riesgos } \\
\text { descritos para el feto } \\
\text { humano }\end{array}$ & $\begin{array}{l}\text { Estudios en animales no han } \\
\text { mostrado riesgo teratógeno; no hay } \\
\text { estudios controlados en mujeres } \\
\text { embarazadas o bien los estudios } \\
\text { en animales han mostrado efectos } \\
\text { secundarios no confirmados } \\
\text { en mujeres embarazadas. } \\
\text { Generalmente, se acepta el uso de } \\
\text { estos medicamentos. }\end{array}$ & $\begin{array}{l}\text { Amoxicilina/Ac. clavulánico, } \\
\text { ampicilina/ sulbactam, } \\
\text { cefalosporinas, cimetidina, } \\
\text { eritromicina, clindamicina, } \\
\text { clotrimazol, metronidazol, } \\
\text { nitrofurantoína, insulina, ranitidina, } \\
\text { dimenhidrinato, cetirizina, } \\
\text { (ketoprofeno, es B 1er y } 2 \text { do T; D en } \\
\text { el 3er T). }\end{array}$ \\
\hline Categoría C & $\begin{array}{l}\text { No puede descartarse } \\
\text { riesgo fetal }\end{array}$ & $\begin{array}{l}\text { Estudios sobre animales han } \\
\text { mostrado efectos secundarios fetales } \\
\text { (teratógenos, embriocidas u otros), } \\
\text { sin que existan estudios controlados } \\
\text { en mujeres gestantes; o no se } \\
\text { dispone de estudios en animales } \\
\text { ni en mujeres. Estos fármacos se } \\
\text { utilizarán solo en caso de que los } \\
\text { beneficios superen a los potenciales } \\
\text { riesgos fetales. }\end{array}$ & $\begin{array}{l}\text { Amikacina, gentamicina, } \\
\text { ciprofloxacina, claritromicina, } \\
\text { cotrimoxazol, isoniazida, ambroxol, } \\
\text { dexametasona, dextrometorfano, } \\
\text { (codeína C 1er y 2do T; D 3er T, y } \\
\text { en dosis prolongadas); diclofenaco, } \\
\text { ketorolaco; (celecoxib y naproxeno } \\
\text { C 1er y 2do T; D 3er T); (AAS, es C } \\
\text { 1er y 2do T; D 3er T ó altas dosis); } \\
\text { (captopril C 1er T; D 2do y 3er T). }\end{array}$ \\
\hline
\end{tabular}




\begin{tabular}{|c|c|c|c|}
\hline Categoría & Seguridad & Descripción & Fármacos de uso frecuente \\
\hline Categoría D & $\begin{array}{l}\text { Hay indicios de riesgo } \\
\text { fetal }\end{array}$ & $\begin{array}{l}\text { A veces los posibles beneficios del } \\
\text { fármaco pueden ser aceptables a } \\
\text { pesar del riesgo (por ejemplo, si el } \\
\text { fármaco se requiere para tratar una } \\
\text { enfermedad que amenaza la vida, o } \\
\text { una enfermedad grave para la que } \\
\text { no pueden usarse otros fármacos } \\
\text { más seguros). }\end{array}$ & $\begin{array}{l}\text { Alprazolam, diazepam, } \\
\text { carbamazepina, estreptomicina, } \\
\text { fenitoína, fenobarbital. }\end{array}$ \\
\hline Categoría X & $\begin{array}{l}\text { Contraindicados en el } \\
\text { embarazo }\end{array}$ & $\begin{array}{l}\text { Tanto los estudios en animales } \\
\text { como en humanos han puesto de } \\
\text { manifiesto evidentes riesgos para } \\
\text { el feto que superan claramente } \\
\text { cualquier beneficio. }\end{array}$ & $\begin{array}{l}\text { Atorvastatina, clomifeno, } \\
\text { anticonceptivos orales combinados } \\
\text { y hormonoterapia de reemplazo, } \\
\text { desogestrel, etinilestradiol, } \\
\text { dihidroergotamina, isotretinoína, } \\
\text { warfarina, vitamina A en altas dosis. }\end{array}$ \\
\hline
\end{tabular}

Actualmente se considera que el sistema no es ideal porque en lugar de simplificar su asesoría, deja en el médico la responsabilidad de interpretar la información de la categoría con base al momento de la exposición, dosis y vía de administración. Hay algunos errores como el de atribuir a los anticonceptivos orales como de categoría $X$ con base en la falta de beneficio de los ACOs en el embarazo, lo cierto es que estas hormonas no tienen ningún riesgo teratogénico con su exposición inadvertida ${ }^{(2)}$.

En el año 2008 la FDA reconoce las limitaciones y ha propuesto nuevas reglas para etiquetar los fármacos en donde considera, evaluación clínica, exposición inadvertida y riesgo disponible ${ }^{(7)}$.

En 2015, la FDA continúa eliminando gradualmente las categorías anteriores y exige la información de estudios en humanos y animales disponibles de las reacciones adversas maternas o fetales conocidos o potenciales, y los ajustes necesarios de la dosis durante el embarazo y el puerperio ${ }^{(3)}$. Desde junio del 2015 exige el etiquetado de los medicamentos con información pormenorizada de los riesgos en la gestación tanto para pacientes como para médicos ${ }^{(11,12)}$.

\section{Medicación específica en el embarazo}

Anemia y estados carenciales:

No hay riesgos para el feto con la ingesta de hierro, pero hay que considerar que su administración en el primer trimestre no se hace necesaria debido a que, durante este periodo, no aumentan las necesidades fisiológicas. Luego hay que administrar suplementos en una cantidad de 30 a $60 \mathrm{mg}$, considerando que para una absorción de $6 \mathrm{mg}$ diarios, se logra con $30 \mathrm{mg} /$ día de hierro elemental es decir con $250 \mathrm{mg}$ de gluconato ferroso, $150 \mathrm{mg}$ de sulfato ferroso, o $90 \mathrm{mg}$ de fumarato ferroso ${ }^{(13,14)}$.

\section{Patología digestiva}

Náuseas y vómitos:

El mecanismo principal de los antihistamínicos, en el tratamiento de las náuseas y los vómitos del embarazo, es la inhibición directa de histamina en el receptor H1. El más recomendado es la doxilamina. Se indica la combinación doxilamina-piridoxina en el tratamiento farmacológico inicial de las náuseas del embarazo. Si persiste se sugiere agregar difenhidramina 25 a $50 \mathrm{mg}$ VO cada seis horas o meclizina $25 \mathrm{mg}$ VO cada seis horas. Si los síntomas no mejoran, se sugiere agregar un antagonista de la dopamina (proclorperazina, metoclopramida) ${ }^{(15,16)}$. Todos ellos seguros en el primer trimestre del embarazo.

El dimenhidrinato es el antihistamínico más usado en la gestante. Es considerado de categoría B por la FDA, no se han realizado estudios en humanos, no se ha establecido la seguridad de su uso durante la gestación pero tampoco se reportan alteraciones por su frecuente uso en el primer trimestre de la gestación.

\section{Reflujo gastrointestinal:}

Se recomienda modificar estilos de vida, como ingerir comidas ligeras y frecuentes, evitar grasas, picantes, café, no acostarse inmediatamente tras la ingesta y elevar la cabecera de la cama. Si estas medidas no son eficaces, considerar el tratamiento farmacológico. Los antiácidos como hidróxidos de magnesio y aluminio, sucralfato, y almagato suelen ser la primera alternativa, aún así no se aconseja usarlos en los 3 primeros meses y por tiempo prolongado. La ranitidina y el omeprazol se 
consideran como seguros ${ }^{(17)}$. Otro estudio por Pasternak (18) que incluyó lansoprazol, esomeprazol y pantoprazol no demostró incremento el riesgo de defectos congénitos.

Estreñimiento:

El tratamiento de primera elección es el aumento de fibra dietética y líquidos. El uso de laxantes como hidróxido de magnesio, lactulosa, o bisacodilo tienen un buen perfil de seguridad debido a su absorción mínima.

Diarrea aguda:

Inicialmente, medidas generales tales como hidratación y dieta apropiada. La loperamida no fue teratogénica en estudios con animales, pero los datos en humanos son contradictorios ${ }^{(19)}$. La terapia con antibióticos rara vez es necesaria debido a su etiología mayormente viral que suele autolimitarse.

\section{Infecciones}

Uso de antibióticos en gestación:

Tienen un buen perfil de seguridad en mujeres embarazadas las cefalosporinas, penicilinas, eritromicina (excepto estolato), azitromicina y la clindamicina. Los aminoglucósidos son relativamente seguros, pero conllevan el riesgo de ototoxicidad y nefrotoxicidad fetal y materna. Aunque la eritromicina y azitromicina tienen buenos perfiles de seguridad, la claritromicina, otro macrólido, ha producido resultados adversos del embarazo en estudios con animales ${ }^{(20,21)}$, La doxiciclina se evita debido a que otras tetraciclinas se han asociado con la supresión transitoria del crecimiento óseo y con la tinción de los dientes en desarrollo ${ }^{(22)}$, pero los datos disponibles no muestran efectos teratogénicos de doxiciclina (23,24). Las fluoroquinolonas se evitan generalmente durante el embarazo y la lactancia, ya que son tóxicos para el desarrollo del cartílago en estudios experimentales en animales. Sin embargo, ni los efectos adversos sobre el cartílago ni un aumento de malformaciones congénitas del uso durante el embarazo humano se ha documentado ${ }^{(25)}$.

Trimetoprim se evita generalmente en el primer trimestre debido a que es un antagonista del ácido fólico (26,27), ha provocado el desarrollo anormal de embriones en animales pero no en seres humanos. Las sulfonamidas no parecen implicar un riesgo significativo, aunque existe una preocupación teórica sobre la hiperbilirrubinemia en el recién nacido prematuro si se administra cerca del parto (8). Una revisión sistemática no encontró ningún caso de kernicterus asociado con el uso materno de sulfonamidas durante el embarazo o la lactancia ${ }^{(28)}$.

Tuberculosis:

El régimen en gestantes se basa en tres fármacos: Isoniacida, rifampicina y etambutol. Hay regiones en donde existe resistencia a la isoniacida, entonces se agrega pirazinamida. Las gestantes que reciben isoniacida deben tomar $25 \mathrm{mg}$ diarios de piridoxina para reducir sus efectos hepatotóxicos y de neuropatía periférica. Están contraindicadas la estreptomicina y, la kanamicina ${ }^{(2,8)}$.

Resfrío común:

Los medicamentos sintomáticos son inocuos en la gestación si se administran por un corto plazo. El paracetamol es el de primera elección y es carente de riesgos. Si la rinorrea es una molestia considerable podemos usar bromuro de ipratropio y aerosol nasal de cromoglicato. Si se precisa un antitusígeno, utilizar dextrometorfano. El uso de fármacos para la garganta a base de cloruro de cetilpiridinio no tiene riesgo. Los aerosoles anestésicos, lidocaína tópica son de categoría $B$ en el embarazo y la benzocaína tópica es de categoría C. No se ha asociado un mayor riesgo de anomalías con la mayoría de los antihistamínicos comúnmente utilizados, como la clorfeniramina. Sin embargo la terfenadina, astemizole, fenilpropanolamina y fenilefrina en el primer trimestre se han relacionado en algunos estudios con riesgo de teratogenicidad ${ }^{(29)}$, por lo que si es necesario descongestionar se deben recurrir a los aerosoles nasales tópicos que representan una dosis más baja para el feto que la medicación sistémica.

El uso de la codeína como antitusígeno no ha reportado aumento del RR de malformaciones pero en dosis excesiva cerca del parto puede causar adicción y síntomas de abstinencia en el recién nacido. El yoduro de potasio como componente de los expectorantes debe evitarse porque cruza la barrera placentaria y puede producir bocio fetal $(2,29)$.

\section{Infecciones urinarias:}

En general se acepta que las penicilinas, cefalosporinas, aztreonam y fosfomicina son seguros en el embarazo. Nitrofurantoína se utiliza mucho pero existen algunas preocupaciones ${ }^{(30)}$. En un estudio de Nordeng ${ }^{\left({ }^{(31)}\right.}$ con 1334 mujeres expuestas en el primer trimestre no se informaron aumentos en las malformaciones. Los aminoglucósidos se han asociado con ototoxicidad después de la exposición fetal prolongada, y por lo tanto debe evitarse a menos que no haya alternativas de tratamiento.

En enterobacterias productoras de betalactamasas de espectro extendido (BLEE), el meropenem, ertapenem, o doripenem son los carbapenémicos más seguros en el embarazo ${ }^{(32,33)}$.

Tricomoniasis:

El metronidazol es usado con frecuencia sin efectos tóxicos sobre el feto. Aunque algunos podrían cuestionar por sus efectos mutagénicos sobre procariotas. En el meta análisis de Caro-Patón ${ }^{(34)}$ y en el estudio de Catherine A. Koss ${ }^{(35)}$ no se encontró ninguna relación entre la exposición al metronidazol durante el primer trimestre del embarazo 
y los defectos de nacimiento. El tinidazol, un derivado imidazólico con actividad antiprotozoica y antianaeróbica, atraviesa la barrera placentaria y no debe ser administrado a pacientes embarazadas en el primer trimestre.

\section{Vaginosis bacteriana:}

Se ha vinculado con aborto espontáneo, parto prematuro, RPM y corioamnionitis por lo que se considera importante el tratamiento cuando es correctamente diagnosticado. El esquema de clindamicina y metronidazol por vía oral durante 7 días ${ }^{(8)}$ que se recomienda en la gestante, no tiene efectos nocivos en el feto, con las particularidades del metronidazol mencionados anteriormente.

\section{Candidiasis vaginal:}

Se recomienda fármacos en crema tipo azoles durante 7 días. Clotrimazol al $1 \%$, miconazol al $2 \%$, butoconazol al $2 \%$ y terconazol al $0.4 \%$, no han reportado efectos teratogénicos. El fluconazol e itraconazol están contraindicados en la gestante por los reportes de malformaciones congénitas bien documentados ${ }^{(8)}$.

\section{Patología neurológica y psiquiátrica}

\section{Epilepsia:}

Está comprobado el riesgo teratógeno de las drogas antiepilépticas, aunque también se relacionan con las altas dosis o con el uso combinado de 3 o más drogas. Se conoce el síndrome por difenilhidantoina (anomalías cráneo-faciales, retraso del crecimiento, retardo mental y defectos en los miembros). El índice de malformaciones mayores es aumentado por el valproato de sodio en 4 a 8 veces, con la difenilhidantoina y el fenobarbital en 2 a 3 veces; la lamotrigina lo reportan con el menor índice de alteraciones. Se recomienda reducir dosis con control clínico estrecho y monitorizar los niveles séricos de anticonvulsivantes ${ }^{(36-39)}$.

Para pacientes con epilepsia idiopática que han estado libres de ataques durante 2 años y que tienen un electroencefalograma (EEG) normal, puede ser seguro intentar una prueba de retiro del medicamento antes del embarazo (2).

\section{Embarazada con migraña:}

El paracetamol, narcóticos y antieméticos son de primera elección. los antiinflamatorios no esteroideos (AINE) son permitidos hasta la semana 30 . El sumatriptan se considera seguro en pacientes con síntomas moderadoseveros que no responden a otros analgésicos, no se han reportado alteraciones congénitas o aumento de abortos, es un fármaco que actúa estimulando los receptores de la serotonina, de esta manera produce una constricción de los vasos sanguíneos craneales que se encuentran distendidos e inflamados durante el ataque de migraña.
Insomnio y ansiedad

Uso de benzodiacepinas:

El riesgo teratógeno es pequeño ${ }^{(40)}$, por ejemplo, puede aumentar el riesgo de fisura oral de 6 de cada 10.000 nacimientos (riesgo normal en la población) a 11 de cada 10.000 nacimientos. Hay mayor riesgo de aborto espontáneo (OR 2), y parto prematuro (OR 1.5). La administración al final del embarazo puede provocar en el neonato: Hipotermia, Apgar bajo, apnea, letargo, hipotonía o hipertonía, inquietud, temblor, hiperreflexia, irritabilidad, mala alimentación, vómitos, diarrea ${ }^{(2,41)}$.

\section{Otras patologías}

\section{El dolor en la gestante}

Paracetamol en la gestante: Es de primera elección como antipirético y analgésico. Algunos estudios lo relacionan con el trastorno por déficit de atención e hiperactividad (TDAH) y criptorquidia ${ }^{(42,43,44)}$, varios estudios posteriores como el realizado por Ystrom et al. ${ }^{(45)}$ en el 2017 con los ajustes estadísticos correspondientes informaron que solo había un riesgo de TDAH cuando se usa por más de 29 días, nuestras pacientes requieren estos analgésicos por corto tiempo, de manera que no hay mayor riesgo ${ }^{(46)}$.

Uso de los antiinflamatorios no esteroideos (AINE): Ibuprofeno, naproxeno, diclofenaco y piroxicam, no se consideran teratógenos pero pueden tener efectos adversos en el feto cuando se administran en el tercer trimestre. Los riesgos y beneficios para el tratamiento del dolor o la fiebre dependen de la dosis, la edad gestacional, y la duración de la terapia. Algunos reportes lo relacionan con el embarazo prolongado y el sangrado post parto. La indometacina y el naproxeno pueden provocar dificultad respiratoria en niños prematuros por el cierre prematuro del ductus arterioso. Un trabajo con ibuprofeno mostró que sí está asociado a abortos por lo que se recomienda no usar AINE cuando hay antecedentes de abortos recurrentes $(47,48)$.

Reacciones alérgicas:

Los antihistamínicos se clasifican en generaciones: De primera generación (doxilamina, dimenhidrinato, clorfenamina), de segunda generación (cetirizina, terfenadina, loratadina), y tercera generación (levocetirizina, desloratadina). La cetirizina y loratadina se consideran de primera elección. La seguridad de los antihistamínicos se afirmó en un metanálisis que examinó la asociación con malformaciones mayores ${ }^{(49)}$. Se concluyó que al parecer tienen un efecto protector sobre el riesgo de malformaciones (OR 0.76).

Los corticoides intranasales son el tratamiento 
recomendado para la rinitis alérgica, debido a su baja absorción sistémica.

Asma bronquial en la gestante:

El manejo de la paciente con asma es generalmente igual que en la no gestante, se prefiere el uso de la medicación inhalatoria que la vía oral o parenteral ajustándose a la severidad y características del cuadro clínico. El salbutamol, el cromoglicato y la teofilina son seguros en la gestante. La terbutalina se ha utilizado ampliamente en el tratamiento del trabajo de parto prematuro, es más rápido en el inicio, tiene una duración de acción más prolongada que la epinefrina, por lo que es de elección en la gestante (2). En asma refractaria al tratamiento con estas drogas o en exacerbaciones del cuadro se tiene que recurrir a corticoides orales, drogas sin mayor riesgo en la gestante.

Todos los corticoides atraviesan la placenta en diferente magnitud. Cuando administramos prednisona o prednisolona a una gestante la concentración del compuesto activo en el feto es inferior al $10 \%$ de la madre, por ello son de elección en el tratamiento del asma. Cuando se desean efectos esteroideos en el feto para acelerar la madurez pulmonar, se utilizan betametasona y dexametasona porque atraviesan la placenta en gran concentración. Un metanálisis publicado en el año $2000{ }^{(50)}$, describe un odds ratio (OR) de 3.0 para el labio leporino y / o el paladar hendido, sin embargo en el 2011, un estudio de cohorte en Dinamarca ${ }^{(51)}$ con 52,000 gestantes expuestas a corticosteroides durante el embarazo en el primer trimestre no identificó riesgo fisuras orofaciales, el OR fue de 1,05 .

Diabetes mellitus:

Los hipoglucemiantes orales (sulfonilureas y biguanidas) no se recomiendan, debido a que se han asociado a efectos teratogénicos en algunas especies animales, aunque sí se puede continuar con la metformina. Se recomienda el uso de insulina NPH que es de efectividad y seguridad comprobada por décadas, y realizar los ajustes y correcciones de dosis de acuerdo a los resultados del automonitoreo de la glicemia.

Hipertensión en la gestante:

El labetalol, nifedipina, hidralazina y la metildopa son seguras en el embarazo ${ }^{(52,53,54)}$. Hay que considerar que la metildopa es sólo un agente antihipertensivo suave y tiene un inicio de acción lento (tres a seis horas), muchas mujeres no van a lograr los objetivos de presión arterial con este agente oral, puede molestar su efecto sedante en dosis altas ${ }^{(55,56)}$.

Vacunas:

Las vacunas contra tétanos, difteria, gripe, hepatitis A y $B$, neumococo se recomiendan en pacientes embarazadas sobre todo si existe un riesgo de infección. Se deben evitar las vacunas de virus vivos. La vacuna triple contra el sarampión, la rubeola y la parotiditis está contraindicada en una gestante.

Asesoría en caso de exposición a teratógenos: Una pregunta reiterativa de los pacientes que han ingerido un fármaco sospechoso de provocar malformaciones es, si efectivamente tendrán alteraciones genéticas sus hijos al nacer. Generalmente las mujeres que llegan con esta inquietud, están mal informadas respecto al verdadero riesgo del fármaco expuesto, un estudio revela que, pensaban que tenían un $25 \%$ de riesgo de anomalías fetales, es decir el riesgo parecido con la talidomida. La información errada puede provocar la interrupción de un embarazo deseado.

La forma en que se presenta la información afecta la percepción del riesgo. Si reciben información negativa, por ejemplo una probabilidad de 1 a $3 \%$ de tener un hijo malformado, tiene mayor probabilidad de percibir un riesgo exagerado que las mujeres a las que se les da una información positiva, es decir, una probabilidad de 97 a 99 $\%$ de tener un hijo sin malformaciones ${ }^{(57)}$.

Hay que considerar que los fármacos prescritos con mayor frecuencia pueden administrarse con relativa seguridad durante el embarazo. Para los escasos medicamentos que se consideran teratógenos, la asesoría debe subrayar el riesgo relativo. Todas las mujeres tienen un riesgo aproximado de $3 \%$ de tener un recién nacido con algún defecto congénito. Aunque la exposición a un teratógeno confirmado puede elevar este riesgo, casi siempre aumenta solo en 1 a $2 \%$, en el peor de los casos al doble o triple. También debe sopesar presentarse el riesgo frente al beneficio. Sin tratamiento algunas enfermedades imponen una amenaza más grave para la madre y el feto que cualquier riesgo teórico de la exposición farmacológica ${ }^{(8)}$.

\section{REFERENCIAS BIBLIOGRÁFICAS}

1. U.S. Department of Health and Human Services, Food and Drug Administration, Center for Drug Evaluation and Research, Center for Biologics Evaluation and Research. Reviewer guidance: evaluating the risks of drug exposure in human pregnancies.

2. Niebyl J, Weber R, and Briggs G. Drugs and Environmental Agents in Pregnancy and Lactation: Teratology, Epidemiology. En: Gabbe SG, Niebyl JR. Niebyl, Simpson JL,et al, editores. Obstetrics: Normal and Problem Pregnancies.7ma ed. Philadelphia: Elsevier; 2017, p.136-158

3. Werler MM, Mitchell AA, Hernandez-Diaz S, Honein MA. Use of over-the-counter medications during pregnancy. Am J Obstet Gynecol. 2005;193(3):771-7.

4. Lockwood C, Magriples U. Initial prenatal assessment and first trimester prenatal care. 2014, UpToDate, Waltham, MA[online] Disponible en: http://www.uptodate.com/ 
contents/initial-prenatal-assessment-and-first-trimesterprenatal

5. Pietrantoni M. Intoxicaciones durante el embarazo. In: Gleicher. Tratamiento de las complicaciones clínicas del embarazo. $3^{a}$ ed. Buenos Aires. Panamericana. 2000: 343360

6. Cuéllar, Santiago, Núñez Manuel y Raposo, Carlos. Uso de medicamentos en embarazo. En: Administración de medicamentos en circunstancias especiales. Ed. Barcelona. 2000, p.2-44. Disponible en: http://www.sld.cu/galerias/ $\mathrm{pdf} /$ sitios/genetica/medicamentos_y_embarazo.pdf

7. Clayton-Smith J, Donnai D. Human malformations. En: Rimoin DL, Connor JM, Pyeritz RE, editores. Emery and Rimoin's Principles and Practice of Medical Genetics. 3ra ed. New York: Churchill Livingstone; 1996, p. 383

8. Cunningham FG, Leveno KJ, Blom SL, Haulth JC, Gilstrap LC, Wenstrom KD. Obstetricia de Williams. 24th ed. Mexico: McGraw Hill; 2015. p240-255

9. McKeigue PM, Lamm SH, Linn S, Kutcher JS. Bendectin and birth defects: I. A meta-analysis of the epidemiologic studies. Teratology. 1994;50(1):27-37.

10. Orueta Sánchez R, López Gil MJ. Manejo de fármacos durante el embarazo. Inf Ter Sist Nac Salud. 2011; 35 (4): 107-113.

11. FDA/CDER SBIA CHRONICLES. Drugs in Pregnancy and Lactation: Improved Benefit-Risk Information. Disponible en. http://www.fda.gov/downloads/Drugs/Development ApprovalProcess/ SmallBusinessAssistance/UCM431132.pdf.

12. Gee RE, Wood SF, Schubert KG. Women's health, pregnancy, and the US Food and Drug Administration. Obstet Gynecol. 2014; 123(1):161-5.

13. Pannone R, Cabrera S, Sosa L. Fármacos en el embarazo y la lactancia. Sitio medico [online] www.sitiomedico.org/artnac/2002/06/32.htm

14. Agirrezabala JR. Medicamentos y embarazo: motivos de consulta frecuentes. INFAC. España. 2005;13(1):1-6. Disponible en: http://www.osakidetza.euskadi.net/

15. Magee LA, Mazzotta P, Koren G. Evidence-based view of safety and effectiveness of pharmacologic therapy for nausea and vomiting of pregnancy (NVP). Am J Obstet Gynecol. 2002;186(5):S256-S61.

16. Seto A, Einarson T, Koren G. Pregnancy outcome following first trimester exposure to antihistamines: meta-analysis. Am J Perinatol. 1997;14(03):119-24.

17. Ruigómez A, Rodriguez LAG, Cattaruzzi C, Troncon MG, Agostinis L, Wallander $M-A$, et al. Use of cimetidine, omeprazole, and ranitidine in pregnant women and pregnancy outcomes. . Am J Epidemiol. 1999;150(5):476-81.

18. Pasternak B, Hviid A. Use of proton-pump inhibitors in early pregnancy and the risk of birth defects. N Engl J Med. 2010;363(22):2114-23.

19. www.reprotox.org (Accessed on September 20, 2012).

20. Lin KJ, Mitchell AA, Yau WP, et al. Safety of macrolides during pregnancy. Am J ObstetGynecol 2013; 208:221.e1.

21. Cooper WO, Hernandez-Diaz S, Arbogast PG, et al. Antibiotics potentially used in response to bioterrorism and the risk of major congenital malformations. PaediatrPerinatEpidemiol 2009; 23:18.

22. Cross R, Ling C, Day NP, et al. Revisiting doxycycline in pregnancy and early childhood--time to rebuild its reputation? Expert Opin Drug Saf 2016; 15:367
23. Cooper WO, Hernandez-Diaz S, Arbogast PG, Dudley JA, Dyer SM, Gideon PS, et al. Antibiotics potentially used in response to bioterrorism and the risk of major congenital malformations. Paediatr Perinat Epidemiol. 2009;23(1):1828

24. Cross R, Ling C, Day NP, McGready R, Paris DH. Revisiting doxycycline in pregnancy and early childhood-time to rebuild its reputation?. Expert Opin Drug Saf. 2016;15(3):367-82.

25. Bar-Oz B, Moretti ME, Boskovic R, O’Brien L, Koren G. The safety of quinolones-a meta-analysis of pregnancy outcomes. Eur J Obstet Gynecol Reprod Biol. 2009;143(2):758.

26. Hernández-Díaz S, Werler MM, Walker AM, Mitchell AA. Folic acid antagonists during pregnancy and the risk of birth defects. N Engl J Med. 2000;343(22):1608-14.

27. Hernández-Díaz S, Werler M, Walker A, Mitchell A. Neural tube defects in relation to use of folic acid antagonists during pregnancy. Am J Epidemiol. 2001;153(10):961.

28. Forna F, McConnell M, Kitabire FN, Homsy J, Mermin J, Weidle PJ. Systematic review of the safety of trimethoprimsulfamethoxazole for prophylaxis in HIV-infected pregnant women: implications for resource-limited settings. AIDS Rev. 2006; 8(1):24-36.

29. Yau W-P, Mitchell AA, Lin KJ, Werler MM, Hernandez-Diaz S. Use of decongestants during pregnancy and the risk of birth defects. Am J Epidemiol. 2013;178(2):198-208.

30. Crider KS, Cleves MA, Reefhuis J, Berry RJ, Hobbs CA, Hu DJ. Antibacterial medication use during pregnancy and risk of birth defects: National Birth Defects Prevention Study. Arch Pediatr Adolesc Med. 2009;163(11):978-85.

31. Nordeng H, Lupattelli A, Romøren M, Koren G. Neonatal outcomes after gestational exposure to nitrofurantoin. Obstetrics \& Gynecology. 2013;121(2, PART 1):306-13.

32. Ho P-l, Yip K-S, Chow K-H, Lo JY, Que T-L, Yuen K-y. Antimicrobial resistance among uropathogens that cause acute uncomplicated cystitis in women in Hong Kong: a prospective multicenter study in 2006 to 2008 Diagn Microbiol Infect Dis. 2010;66(1):87-93.

33. Rodríguez-Baño J, Alcalá JC, Cisneros JM, Grill F, Oliver A, Horcajada JP, et al. Community infections caused by extended-spectrum B-lactamase-producing Escherichia coli. Arch Intern Med. 2008;168(17):1897-902.

34. Caro-Patón T, Carvajal A, de Diego IM, Martín-Arias LH, Requejo AA, Pinilla ER. Is metronidazole teratogenic? A meta-analysis. Br J Clin Pharmacol. 1997;44(2):179-82.

35. Koss CA, Baras D, Lane SD, Aubry R, Marcus M, Markowitz LE, et al. Investigation of Metronidazole Use during Pregnancy and Adverse Birth Outcomes. Antimicrob Agents Chemother. 2012; 56(9): 4800-4805.

36. Brees Mark H, Berkow Robert. Manual Merck; 1999:20282033.

37. Molins A. Antiepileptic drugs in the elderly, pregnant women, children and in systemic disorders. Rev Neurology. 2000; 30(9): 865-872.

38. ACOG Committee on Practice Bulletins--Obstetrics. ACOG Practice Bulletin: Clinical management guidelines for obstetrician-gynecologists number 92, April 2008. Use of psychiatric medications during pregnancy and lactation. Obstet Gynecol 2008; 111:1001.

39. Campbell E, Kennedy F, Russell A, Smithson W, Parsons L, Morrison $\mathrm{P}$, et al. Malformation risks of antiepileptic drug 
monotherapies in pregnancy: updated results from the UK and Ireland Epilepsy and Pregnancy Registers. J Neurol Neurosurg Psychiatry. 2014; 85(9): 1029-34.

40. Dolovich LR, Addis A, Vaillancourt JR, Power JB, Koren G, Einarson TR. Benzodiazepine use in pregnancy and major malformations or oral cleft: meta-analysis of cohort and case-control studies. BMJ. 1998;317(7162):839-43.

41. Wikner BN, Stiller CO, Bergman U, Asker C, Källén B. Use of benzodiazepines and benzodiazepine receptor agonists during pregnancy: neonatal outcome and congenital malformations. Pharmaco epidemiol Drug Saf. 2007;16(11):1203-10.

42. Liew Z, Ritz B, Rebordosa C, Lee P-C, Olsen J. Acetaminophen use during pregnancy, behavioral problems, and hyperkinetic disorders. JAMA Pediatr. 2014;168(4):313-20.

43. Thompson JM, Waldie KE, Wall CR, Murphy R, Mitchell EA, Group AS. Associations between acetaminophen use during pregnancy and ADHD symptoms measured at ages 7 and 11 years. PloS one. 2014;9(9):e108210.

44. Kristensen DM, Hass U, Lesné L, Lottrup G, Jacobsen PR, Desdoits-Lethimonier $C$, et al. Intrauterine exposure to mild analgesics is a risk factor for development of male reproductive disorders in human and rat. Hum Reprod. 2010;26(1):235-44.

45. Ystrom E, Gustavson K, Brandlistuen RE, Knudsen GP, Magnus $P$, Susser E, et al. Prenatal exposure to acetaminophen and risk of ADHD. Pediatrics.2017:e20163840.

46. Feldkamp ML, Meyer RE, Krikov S, Botto LD. Acetaminophen use in pregnancy and risk of birth defects: findings from the National Birth Defects Prevention Study. Obstet Gynecol. 2010;115(1):109-15.

47. Nezvalová-Henriksen K, Spigset O, Nordeng H. Effects of ibuprofen, diclofenac, naproxen, and piroxicam on the course of pregnancy and pregnancy outcome: a prospective cohort study. Br J Obstet Gynecol. 2013;120(8):948-59.

48. Hernandez RK, Werler MM, Romitti P, Sun L, Anderka M. Nonsteroidal antiinflammatory drug use among women and the risk of birth defects. Am J Obstet Gynecol. 2012;206(3):228. e1-. e8.

49. Seto A, Einarson T, Koren G. Pregnancy outcome following first trimester exposure to antihistamines: meta-analysis. Am J Perinatol. 1997;14(03):119-24.

50. Park-Wyllie L, Mazzotta P, Pastuszak A, Moretti ME, Beique L, Hunnisett $L$, et al. Birth defects after maternal exposure to corticosteroids: prospective cohort study and meta-analysis of epidemiological studies. Teratology. 2000;62(6):385-392.

51. Hviid A, Mølgaard-Nielsen D. Corticosteroid use during pregnancy and risk of orofacial clefts. CMAJ. 2011;183(7):796.

52. American College of Obstetricians and Gynecologists,
Task Force on Hypertension in Pregnancy. Hypertension in pregnancy. Report of the American College of Obstetricians and Gynecologists' Task Force on Hypertension in Pregnancy. Obstet Gynecol. 2013; 122:1122.

53. Abalos E, Duley L, Steyn D, Henderson-Smart DJ. Antihypertensive drug therapy for mild to moderate hypertension during pregnancy. Cochrane Database Syst Rev. 2014;(2): CD002252.

54. Magee LA, von Dadelszen P, Rey E, Ross S, Asztalos E, Murphy $\mathrm{KE}$, et al. Less-tight versus tight control of hypertension in pregnancy. N Engl J Med. 2015;372(5):407-17.

55. Peacock WF, Hilleman DE, Levy PD, Rhoney DH, Varon J. A systematic review of nicardipine vs labetalol for the management of hypertensive crises. Am J Emerg Med. 2012;30(6):981-93.

56. Molvi SN, Mir S, Rana VS, Jabeen F, Malik AR. Role of antihypertensive therapy in mild to moderate pregnancyinduced hypertension: a prospective randomized study comparing labetalol with alpha methyldopa. Arch Gynecol Obstet. 2012;285(6):1553-62.

57. Jasper J, Goel R, Einarson A, Gallo M, Koren G. Effects of framing on teratogenic risk perception in pregnant women. Lancet. 2001;358(9289):1237-8.

Fuentes de financiamiento:

Este artículo ha sido financiado por los autores.

Conflictos de interés:

Los autores declaran no tener ningún conflicto de interés.

Correspondencia:

José Sandoval Paredes

Dirección: Calle Rio de Janeiro N145. Jesús María

Teléfono: 999041839

Correo electrónico: jsandovalpar@hotmail.com

Recibido: 08 de abril de 2018

Evaluado: 17 de abril de 2018

Aprobado: 01 de mayo de 2018

(c) La revista. Publicado por Universidad de San Martín de Porres, Perú. (c) By Licencia de Creative Commons Artículo en acceso abierto bajo términos de Licencia Creative Commons Atribución 4.0 Internacional. (http://creativecommons.org/licenses/by/4.0/)

ORCID iDs

José Sandoval Paredes

http: / / orcid.org/0000-0002-4073-5699 